\title{
Green Functions in Coordinate Space for Gauge Bosons at Finite Temperature
}

\author{
H. Arthur Weldon \\ Department of Physics, West Virginia University, Morgantown, West Virginia, 26506-6315
}

(October 29, 2018)

\begin{abstract}
The thermal Green function, $\mathcal{D}^{\mu \nu}(x)$, for free, massless gauge bosons is computed exactly in a variety of gauges (Feynman, covariant, Coulomb, and Landshoff-Rebhan). At large temporal separations it falls exponentially. At large spatial separations it falls like $T / r$. In contrast, the zero-temperature propagator falls quadratically in both regimes, being proportional to $1 /\left(t^{2}-r^{2}\right)$.

11.10.Wx, 12.38.Mh, 14.70.Bh
\end{abstract}

\section{INTRODUCTION}

Although quantum field theories are formulated in four-dimensional coordinate space, calculations are almost always performed in four-dimensional momentum space. One major exception to the preference for momentum space is non-equilibrium field theory. Without equilibrium there is no invariance under time-translation and often no invariance under spatial translation. The dynamics of the density operator $\varrho$ controls the evolution. For gauge bosons the time-ordered propagator is

$$
\mathcal{D}_{11}^{\mu \nu}(x, y)=-i \operatorname{Tr}\left(\varrho T\left(A^{\mu}(x) A^{\nu}(y)\right)\right) .
$$

In non-equilibrium the correlations depend not just on the separation $x-y$ but also on the effective age of the system $x+y$. For situations in which the dynamical evolution depends rapidly on $x-y$ and slowly on $x+y$, it is standard to Fourier transform from the separation $x-y$ to the conjugate four-momentum $K$. The transformed propagator is then a function of $K$ and of $x+y$. It has long been known [1 4 how to use the Schwinger-Dyson equation for the non-equilibrium propagator to extract a kinetic equation for the non-equilibrium distribution function $f(K)$ that depends on $x+y$. This approach has been extended to modern field theories containing gauge bosons particularly for QCD 5 ,9].

In ordinary vacuum field theory, or zero-temperature field theory, the density operator is $\varrho=|0\rangle\langle 0|$. Translation invariance is automatic and calculations are almost always performed in momentum space. In momentum space the time-ordered propagator for free gauge bosons in the Feynman gauge is $D_{11}^{\mu \nu}(K)=-g^{\mu \nu} /\left(K^{2}+i \eta\right)$. This can easily be Fourier transformed to give the coordinatespace propagator

$$
\left.\mathcal{D}_{11}^{\mu \nu}(x)\right|_{T=0}=\frac{-i}{4 \pi^{2}} \frac{g^{\mu \nu}}{x^{2}-i \epsilon} .
$$

Even though for fixed $t$ this falls like $1 / r^{2}$ as $r \rightarrow \infty$, it does contain the correct Coulomb potential, which comes from the light-cone singularity $\delta(t \pm r) / r$. At large timelike separations the $1 / t^{2}$ behavior causes the electron propagator to have a branch point at the electron mass shell instead of a pole. The $1 / x^{2}$ behavior often leads to processes that are divergent both in the ultraviolet $(x \rightarrow 0)$ and in the infrared $(x \rightarrow \infty)$.

A further consequence of Eq. (1.1) is that at zero temperature the quantum effects of massless particles are quite complicated because the effects are as important in space-like directions as in time-like directions.

\section{Thermal Equilibrium}

For a field theory in an equilibrium heat bath that is uniform in space and constant in time, there is a constant temperature $T$ throughout. The density operator is given by $\varrho=e^{-H / T} / \operatorname{Tr}\left(e^{-H / T}\right)$. The time-ordered propagator has the structure 10 12

$$
\mathcal{D}_{11}^{\mu \nu}(x)=\theta(t) \mathcal{D}_{>}^{\mu \nu}(x)+\theta(-t) \mathcal{D}_{<}^{\mu \nu}(x) .
$$

The thermal Wightman functions is defined by

$$
\mathcal{D}_{>}^{\mu \nu}(x)=-i \sum_{n} e^{-\beta E_{n}} \frac{\left\langle n\left|A^{\mu}(x) A^{\nu}(0)\right| n\right\rangle}{\operatorname{Tr}\left[e^{-\beta H}\right]},
$$

and $\mathcal{D}_{<}(x)=\mathcal{D}_{>}(-x)$. Appendix A summarizes how all other propagators (contour-ordered, retarded, advanced) can be expressed in terms of $\mathcal{D}_{>}(x)$.

The thermal Wightman function satisfies two important conditions: Eq. (1.4) and (1.5) below. Both conditions come from the Heisenberg relation $A^{\mu}(t, \vec{r})=$ $\exp (i H t) A^{\mu}(0, \vec{r}) \exp (-i H t)$. This implies that for complex time the field satisfies $A^{\mu}(t, \vec{r})^{\dagger}=A^{\mu}\left(t^{*}, \vec{r}\right)$. Consequently the Wightman function enjoys the reflection property

$$
\left[\mathcal{D}_{>}^{\mu \nu}(x)\right]^{*}=-\mathcal{D}_{>}^{\nu \mu}\left(-x^{*}\right)
$$

Furthermore, by inserting a complete set of energy eigenstates between the two field operators in Eq. (1.3), it is simple to show that at complex time $\mathcal{D}_{>}^{\mu \nu}(t, \vec{x})$ is analytic in the strip $-\beta \leq \operatorname{Im} t \leq 0$. A similar argument shows that the Wightman function must satisfy the Kubo-Martin-Schwinger relation 13]

$$
\mathcal{D}_{>}^{\mu \nu}(t-i \beta, \vec{r})=\mathcal{D}_{>}^{\nu \mu}(-t, \vec{r}) .
$$


In momentum space the Wightman function is given by in terms of the momentum-space spectral function $\rho^{\mu \nu}(K)$ by

$$
D_{>}^{\mu \nu}(K)=\frac{-i \rho^{\mu \nu}(K)}{1-e^{-\beta k_{0}}},
$$

which is derived in Appendix A. The free spectral function is independent of temperature and only has support at $k_{0}= \pm|\vec{k}|$. Thus only the on-shell particles contribute to the free Wightman function. The coordinate-space Wightman function is the Fourier transform

$$
\mathcal{D}_{>}^{\mu \nu}(x)=\int \frac{d^{4} K}{(2 \pi)^{4}} e^{-i K \cdot x} D_{>}^{\mu \nu}(K) .
$$

As shown in Appendix A, the properties of the spectral function guarantee that $\mathcal{D}_{>}^{\mu \nu}(x)$ will automatically satisfy the reflection condition in Eq. (1.4) and the KMS relation in Eq. (1.5).

The remainder of the paper will perform the computation of Eq. (1.7) for free gauge bosons in various gauges. Sec II deals entirely with the Feynman gauge. The complete result as a function of arbitrary complex time is displayed in Eq. (2.14), but a simpler form is the specialization to real time shown in Eq. (2.15). Sec III performs the calculation in a general covariant gauge and leads to the results summarized in Eqns. (3.6) and (3.7). Sec IV computes the Wightman function in the Coulomb gauge, which is a bit more subtle, and the results are given in Eqns. 4.6), 4.7), 4.10), and (4.11). Sec V contains a comparison of propagators with Bose-Einstein, Fermi-Dirac, and Boltzman statistics. Appendix A provides some general formulas. Appendix B performs the same calculation in the Landshoff-Rebhan quantization scheme 14] in which the physical, transverse gauge fields are thermalized but not the longitudinal and time-like components.

\section{II. $\mathcal{D}_{>}^{\mu \nu}(x)$ IN FEYNMAN GAUGE}

It is easiest to compute the thermal Wightman function in the Feynman gauge, in which the spectral function is proportional to the constant metric tensor $g^{\mu \nu}$ :

$$
\rho^{\mu \nu}(K)=-g^{\mu \nu} 2 \pi \epsilon\left(k_{0}\right) \delta\left(K^{2}\right) .
$$

The thermal Wightman function has the form

$$
\mathcal{D}_{>}^{\mu \nu}(x)=-g^{\mu \nu} \mathcal{D}_{>}(x)
$$

where

$$
\mathcal{D}_{>}(x)=-i \int \frac{d^{4} K}{(2 \pi)^{3}} e^{-i K \cdot x} \frac{\epsilon\left(k_{0}\right) \delta\left(K^{2}\right)}{1-e^{-\beta k_{0}}} .
$$

The scalar function $\mathcal{D}_{>}(x)$ is, in addition, the thermal Wightman function for a spinless field of zero mass. To compute the integral, first perform the integrals over $k_{0}$ and over the angles of $\vec{k}$ to obtain

$$
\mathcal{D}_{>}(x)=\frac{-1}{8 \pi^{2} r} \int_{0}^{\infty} d k f_{k}(r, t),
$$

where the $k$ dependence is contained in the function

$$
f_{k}(t, r) \equiv\left[e^{i k r}-e^{-i k r}\right]\left[\frac{e^{-i k t}}{1-e^{-\beta k}}-\frac{e^{i k t}}{1-e^{\beta k}}\right] .
$$

This function satisfies the conditions

$$
\begin{aligned}
-f_{k}(t, r)^{*} & =f_{k}\left(-t^{*}, r\right) \\
f_{k}(t-i \beta, r) & =f_{k}(-t, r),
\end{aligned}
$$

which guarantee that $\mathcal{D}_{>}(x)$ will satisfy the reflection and KMS conditions given in Eqs. (1.4) and (1.5).

\section{A. Unregularized $\mathcal{D}_{>}(x)$}

For any real value of $t$, except for $t= \pm r$, the rapid oscillations of the integrand as $k \rightarrow \infty$ guarantee convergence. If $t$ has a negative imaginary part then convergence is improved provided the negative imaginary part is smaller in magnitude than $\beta$. Thus in the region

$$
-\beta<\operatorname{Im} t \leq 0 \quad \& \quad t \neq \pm r
$$

the integral is convergent without any regularization. To perform the integration, it is convenient to use the fact that $f_{k}(t, r)$ is an even function of $k$ in order to extend the integration range to the full $k$ axis:

$$
\mathcal{D}_{>}(x)=\frac{-1}{16 \pi^{2} r} \int_{-\infty}^{\infty} d k f_{k}(r, t)
$$

This can now be evaluated by contour integration over complex $k$. The integrand $f_{k}(t, r)$ is finite at $k=0$. It has poles at $k= \pm i 2 \pi n T$, for integer $n \geq 1$.

How the contour should be closed depends on the relative size of $\operatorname{Re} t$ and $r$. For definiteness, let $\operatorname{Re} t>r$. Then $\exp ( \pm i k t)$ is the determining factor. For the term containing $\exp (-i k t)$ the contour should be closed in the lower-half of the complex $k$ plane; for the term $\exp (+i k t)$, the contour should be closed above. Cauchy's theorem gives the result as a sum of the residues of the poles:

$$
\mathcal{D}_{>}(x)=\frac{-i T}{4 \pi r} \sum_{n=1}^{\infty}\left[\left(z_{+}\right)^{n}-\left(z_{-}\right)^{n}\right],
$$

where the variables $z_{ \pm}$are defined by

$$
z_{+}=e^{-2 \pi T(t+r)} \quad z_{-}=e^{-2 \pi T(t-r)} .
$$

This sum is only convergent in the region $\operatorname{Re} t>r$. Performing the sum gives 


$$
\mathcal{D}_{>}(t, r)=\frac{-i T}{4 \pi r}\left[\frac{1}{e^{2 \pi T(t+r)}-1}-\frac{1}{e^{2 \pi T(t-r)}-1}\right] .
$$

This result is valid for all complex $t$ satisfying Eq. (2.4) and is analytic in this region. The poles at $t= \pm r$ and at $t= \pm r-i \beta$ (limit points of the open region) will be shifted slightly when the integration is regularized. It is convenient to express $\mathcal{D}_{>}(x)$ in the alternate form

$$
\mathcal{D}_{>}(t, r)=\frac{-i T}{8 \pi r}[\operatorname{coth}(\pi T(r+t))+\operatorname{coth}(\pi T(r-t))] .
$$

Note that this is an even function of $t$. The regularized $\mathcal{D}_{>}(x)$ will include the domain $t= \pm r$ and will not be even under $t \rightarrow-t$.

Deep time-like region: For $|t|-r \gg 1 / \pi T$, the asymptotic behavior is

$$
\mathcal{D}_{>}(x) \rightarrow \frac{i T}{4 \pi r}\left[e^{-2 \pi T(|t|-r)}-e^{-2 \pi T(|t|+r)}+\ldots\right] .
$$

Deep space-like region: For $r-|t| \gg 1 / \pi T$, the asymptotic behavior is not exponentially small.

$$
\mathcal{D}_{>}(x) \rightarrow \frac{-i T}{4 \pi r}-\frac{i T}{4 \pi r}\left[e^{-2 \pi T(r-|t|)}+e^{-2 \pi T(r+|t|)}\right] .
$$

The dominant term $T / r$ is present in all gauges and will be discussed further.

\section{B. Regularized $\mathcal{D}_{>}(x)$}

The true Wightman function should be analytic in the closed region

$$
-\beta \leq \operatorname{Im} t \leq 0,
$$

which includes the points $t= \pm r$ and $t= \pm r-i \beta$ at which Eq. (2.7) has poles. The problem is that at these values of $t$ the integrand $f_{k}(t, r) \rightarrow 1$ without oscillation as $k \rightarrow \infty$. The simplest way to regularize the integral in a way that will satisfy Eqs. (1.4) and (1.5) is to define

$$
\mathcal{D}_{>}(x)=\frac{-1}{8 \pi^{2}} \int_{0}^{\infty} d k f(t, r) e^{-\epsilon k},
$$

where $\epsilon$ is positive and real. Now the range of integration cannot be extended to negative $k$. However the integral can be evaluated using the relation 16]

$$
\frac{1}{T} \int_{0}^{\infty} d k \frac{e^{-a_{1} k}-e^{-a_{2} k}}{e^{\beta k}-1}=-\psi\left(1+a_{1} T\right)+\psi\left(1+a_{2} T\right),
$$

which holds whenever the real parts of $a_{1}$ and $a_{2}$ are positive. Here $\psi(z)=d \ln \Gamma(z) / d z$. The result is

$$
\begin{aligned}
\mathcal{D}_{>}(x)= & \frac{T}{8 \pi^{2} r}[\psi[\epsilon T+i T(t-r)]-\psi[\epsilon T+i T(t+r)] \\
& -\psi[1+\epsilon T-i T(t-r)]+\psi[1+\epsilon T-i T(t+r)]] .
\end{aligned}
$$

This satisfies the reflection condition Eq. (1.4), which interchanges the first term with the second and interchanges the third term with the fourth. It satisfies the KMS condition (1.5), which interchanges the first term with the third and the second term with the fourth. Using $\psi(z)=\psi(1+z)-1 / z$ this can be written so as to isolate the zero-temperature contribution:

$$
\begin{gathered}
\mathcal{D}_{>}(x)=\frac{T}{8 \pi^{2} r}[\psi[1+T(\epsilon-i(r+t))]-\psi[1+T(\epsilon+i(r+t))] \\
+\psi[1+T(\epsilon-i(r-t))]-\psi[1+T(\epsilon+i(r-t))]] \\
\quad-\frac{i}{4 \pi^{2}} \frac{1}{(r+t-i \epsilon)(r-t+i \epsilon)}
\end{gathered}
$$

This is the complete and general result. It is analytic in the closed region given in Eq. (2.11). The nearest poles to this region are the zero-temperature poles just above the strip at $t= \pm r+i \epsilon$ and the the poles just below the strip at $t= \pm r-i(\beta+\epsilon)$ from the first and fourth term.

Real time: For real values of $t$ one can safely put $\epsilon=0$ in all four $\psi$ functions since their poles are are at least a distance $\pm i \beta$ from the real $t$ axis. When $\epsilon=0$ the psi functions have complex conjugate arguments and can be simplified using [17]

$$
\psi(1-i y)-\psi(1+i y)=\frac{i}{y}-i \pi \operatorname{coth}(\pi y) .
$$

The Wightman function is

$$
\begin{aligned}
\mathcal{D}_{>}(x)= & \frac{-i T}{8 \pi r}[\operatorname{coth}[\pi T(r+t)+\operatorname{coth}[\pi T(r-t)] \\
& +\frac{1}{8 \pi r}[\delta(r+t)-\delta(r-t)] .
\end{aligned}
$$

This, of course, agrees with Eq. (2.8) when $t \neq \pm r$. Note that under $t \rightarrow-t$, the coth terms are symmetric but the Dirac delta terms are antisymmetric. The other Wightman function $\mathcal{D}_{<}(x)$ for real $t$ is

$$
\begin{aligned}
\mathcal{D}_{<}(x)= & \frac{-i T}{8 \pi r}[\operatorname{coth}[\pi T(r+t)+\operatorname{coth}[\pi T(r-t)] \\
& +\frac{1}{8 \pi r}[\delta(r-t)-\delta(r+t)] .
\end{aligned}
$$

Since Eqns. (2.15) and (2.16) hold only for real $t$, one cannot pass from one to the other by the KMS relation.

\section{Time-ordered propagator}

The Wightman function satisfies the homogeneous equation $\square \mathcal{D}_{>}(x)=0$. The time-ordered propagator is

$$
\mathcal{D}_{11}(x)=\theta(t) \mathcal{D}_{>}(x)+\theta(-t) \mathcal{D}_{<}(x),
$$

in which $\mathcal{D}_{<}(t, r)=\mathcal{D}_{>}(-t, r)$. This is a true Green function in that it satisfies the inhomogeneous equation $\square \mathcal{D}_{11}(x)=-\delta^{4}(x)$. Using Eq. 2.14) gives 


$$
\begin{aligned}
\mathcal{D}_{11}(x)= & \frac{T}{8 \pi^{2} r}[\psi[1+T(\epsilon-i(r+t))]-\psi[1+T(\epsilon+i(r+t))] \\
& +\psi[1+T(\epsilon-i(r-t))]-\psi[1+T(\epsilon+i(r-t))]] \\
& +\frac{i}{4 \pi^{2}} \frac{1}{t^{2}-r^{2}-i \epsilon} .
\end{aligned}
$$

This representation has the nice feature that the zerotemperature limit is isolated in the last term. The temperature-dependent terms are each annihilated by the d'Alembertian operator $\square$.

\section{Potential produced by a static charge}

In the limit $r \rightarrow \infty$ at fixed $t$, the Wightman function and the time-ordered propagator have the behavior $\mathcal{D}(x) \rightarrow-i T /(4 \pi r)$. This contribution suggests that at large distance the potential produced by a charge at rest would be temperature-dependent. This inference is incorrect as the following calculation demonstrates.

Let $J^{\mu}(x)$ be a classical current. In the Feynman gauge, $\mathcal{D}_{11}^{\mu \nu}(x)=-g^{\mu \nu} \mathcal{D}_{11}(x)$ and so the classical vector potential is

$$
A_{\mathrm{cl}}^{\mu}(x)=-\int d^{4} x^{\prime} D_{11}\left(x-x^{\prime}\right) J^{\mu}\left(x^{\prime}\right) .
$$

For a point charge $Q$ at rest $J^{0}\left(x^{\prime}\right)=Q \delta^{3}\left(\vec{r}^{\prime}\right)$ and $\vec{J}\left(x^{\prime}\right)=0$. Thus the three-vector potential vanishes and the scalar potential requires integrating over the static charge density:

$$
A_{\mathrm{cl}}^{0}(x)=-Q \int_{-\infty}^{\infty} d t^{\prime} \mathcal{D}_{11}\left(t-t^{\prime}, r\right)
$$

It is convenient to use Eq. (2.17) for the Green function. At large complex $t$ the combination of psi functions falls like $1 / t^{2}$. Thus one can integrate over complex time by integrating over a contour $C$ that is closed in the upper half-plane:

$$
A_{\mathrm{cl}}^{0}(x)=-Q \oint_{C} d t^{\prime} \mathcal{D}_{11}\left(t-t^{\prime}, r\right)
$$

The first and fourth $\psi$ function in Eq. (2.17) are analytic in the upper-half of the complex $t$ plane and make no contribution. The second and third $\psi$ functions have poles in the upper half-plane at $t= \pm r+i(N \beta+\epsilon)$ for $N=1,2,3 \ldots$. These poles all have the same residue (viz. $-i \beta$ ) and their contributions to the potential cancel exactly. Thus the entire potential comes from the zerotemperature term:

$$
A_{\mathrm{cl}}^{0}(x)=-\frac{i Q}{4 \pi^{2}} \oint_{C} d t^{\prime} \frac{1}{\left(t-t^{\prime}\right)^{2}-r^{2}-i \epsilon} .
$$

This is easily integrated and gives the usual Coulomb potential:

$$
A_{\mathrm{cl}}^{0}(x)=\frac{Q}{4 \pi r} .
$$

It is perhaps worth emphasizing that this not a large distance approximation. A more difficult calculation, which does contain temperature-dependence, is the potential due to a point charge moving with constant velocity, where $J^{0}\left(x^{\prime}\right)=Q \delta^{3}\left(\vec{r}^{\prime}-\vec{v} t^{\prime}\right)$.

\section{III. $\mathcal{D}_{>}^{\mu \nu}(x)$ IN COVARIANT GAUGES}

The next case in which to compute the thermal Wightman functions are the general covariant gauges. The tedious regularization performed in Sec II will not be attempted. The result given in Eq. (3.6) and (3.7) is therefore valid for real $t$ not on the light cone.

In a general covariant gauge the time-ordered propagator at zero temperature is

$$
\left.D_{11}^{\mu \nu}(K)\right|_{T=0}=\frac{-g^{\mu \nu}}{K^{2}+i \epsilon}+(1-\xi) \frac{K^{\mu} K^{\nu}}{\left(K^{2}+i \epsilon\right)^{2}} .
$$

The spectral function, extracted using Eq. (A7), is

$$
\rho^{\mu \nu}(K)=2 \pi \epsilon\left(k_{0}\right)\left[-g^{\mu \nu}+(1-\xi) K^{\mu} K^{\nu} \frac{\partial}{\partial k^{2}}\right] \delta\left(K^{2}\right) .
$$

When the thermal Wightman function $\mathcal{D}_{>}^{\mu \nu}(x)$ is expressed in terms of the spectral function using Eq. (1.7) the result is

$$
\mathcal{D}_{>}^{\mu \nu}(x)=-g^{\mu \nu} \mathcal{D}_{>}(x)+(1-\xi) \frac{\partial^{2}}{\partial x_{\mu} \partial x_{\nu}} d_{>}(x),
$$

where $\mathcal{D}_{>}(x)$ with no superscripts is the Wightman function from Sec II and the new function $d_{>}(x)$ is

$$
d_{>}(x)=i \int \frac{d^{4} K}{(2 \pi)^{3}} \frac{e^{-i K \cdot x}}{1-e^{-\beta k_{0}}} \epsilon\left(k_{0}\right) \frac{\partial}{\partial k^{2}} \delta\left(K^{2}\right) .
$$

It will be important later that $\mathcal{D}_{>}^{\mu \nu}(x)$ is not affected if a constant is added to the value of $d_{>}(x)$. The integration over $k_{0}$ and over the angles gives

$$
d_{>}(x)=\frac{-i}{16 \pi^{2}} \int_{0}^{\infty} \frac{d k}{k^{2}} g_{k}(t, r),
$$

where

$$
g_{k}(t, r)=k\left[e^{i k r}+e^{-i k r}\right]\left[\frac{e^{-i k t}}{1-e^{-\beta k}}-\frac{e^{i k t}}{1-e^{\beta k}}\right] .
$$

The function $g_{k}(t, r)$ equals $4 T$ at $k=0$. Consequently the integral in Eq. (3.2) does not converge at $k=0$. However the fact that $\partial g_{k}(t, r) / \partial t$ and $\partial g_{k}(t, r) / \partial r$ both vanish like $k^{2}$ as $k \rightarrow 0$ guarantees that $\partial d_{>}(x) / \partial x_{\mu}$ is finite, which is all that is necessary for Eq. (3.1). Since the behavior at $k=0$ is awkward, it is convenient to subtract a constant from Eq. (3.2) and redefine $d_{>}(x)$ as 


$$
d_{>}(x)=\frac{-i}{16 \pi^{2}} \int_{0}^{\infty} \frac{d k}{k^{2}}\left[g_{k}(t, r)-g_{k}(0,0) \frac{\mu^{2}}{k^{2}+\mu^{2}}\right] .
$$

The integrand now has no singularity at $k=0$. A real parameter $\mu$ has been introduced so that the subtracted integration converges at $k=\infty$ for non-exceptional values of $t$. Since it is not regulated as was done in Sec IIB, it does not converge for $t= \pm r$ or for $t= \pm r-i \beta$.

It is simple to integrate Eq. (3.3). The integrand is even in $k$ and thus the range may be extended to $-\infty$ :

$$
d_{>}(x)=\frac{-i}{32 \pi^{2}} \int_{-\infty}^{\infty} \frac{d k}{k^{2}}\left[g_{k}(t, r)-g_{k}(0,0) \frac{\mu^{2}}{k^{2}+\mu^{2}}\right] .
$$

The integral can be evaluated by closing the contour in the complex $k$ plane and using Cauchy's theorem. How the contour is closed depends upon the relative size of $t$ and $r$. For example, if Re $t>r$ the contour for the terms containing $\exp (-i k t)$ should be closed in the lower halfplane; those containing $\exp (i k t)$ should be closed in the upper half-plane. The integrand of (3.3) is not singular at $k=0$. The poles at $k= \pm i 2 \pi n T$ for $n=1,2,3 \ldots$. give the result

$$
d_{>}(x)=\frac{-i}{16 \pi^{2}} \sum_{n=1}^{\infty}\left(\frac{\left(z_{+}\right)^{n}}{n}+\frac{\left(z_{-}\right)^{n}}{n}\right)
$$

where the constant terms independent of $t$ and $r$ are omitted and $z_{ \pm}$are the same as in Eq. (2.6). The summation displayed is only convergent if the magnitudes of $z_{ \pm}$are less than one: i.e. $\operatorname{Re} t>r$. Performing the sum in this region gives

$$
d_{>}(x)=\frac{i}{16 \pi^{2}}\left[\ln \left(1-z_{+}\right)+\ln \left(1-z_{-}\right)\right] .
$$

This result can now be extended to $\operatorname{Re} t<r$. Since it was not regularized, it is not valid precisely at $z_{+}=1$ or at $z_{-}=1$. The result is not symmetric under $t \rightarrow-t$, but it can be rewritten as

$$
\begin{aligned}
d_{>}(x)= & \frac{i}{16 \pi^{2}} \ln [\sinh (\pi T(r+t)) \sinh (\pi T(r-t))] \\
& -\frac{i T}{8 \pi} t+\text { constant. }
\end{aligned}
$$

The term linear in $t$ and the constant do not have second derivatives and thus do not affect the value of $D_{>}^{\mu \nu}(x)$. The function $d_{>}(x)$ is related to the scalar Wightman function by $\square d_{>}(x)=\mathcal{D}_{>}(x)$. Therefore Eq. (3.1) can be summarized by

$$
\mathcal{D}_{>}^{\mu \nu}(x)=\left(-g^{\mu \nu} \square+(1-\xi) \partial^{\mu} \partial^{\nu}\right) d_{>}(x) .
$$

The form of this in various limits will now be examined.

Deep time-like region: When $|t|-r \gg 1 / \pi T$ the asymptotic behavior of Eq. (3.6) is

$$
d_{>}(x) \rightarrow \frac{-i}{16 \pi^{2}}\left[e^{-2 \pi T(|t|-r)}+e^{-2 \pi T(|t|+r)}\right],
$$

with an irrelevant term linear in $t$ omitted. Consequently all components of $\mathcal{D}_{>}^{\mu \nu}(x)$ fall exponentially as was the case in Feynman gauge.
Deep space-like region: If $r-|t| \gg 1 / \pi T$, then Eq. (3.6) has the behavior

$$
d_{>}(x) \rightarrow \frac{i T r}{8 \pi}-\frac{i}{16 \pi^{2}}\left[e^{-2 \pi t(r-|t|)}+e^{-2 \pi T(r+|t|)}\right],
$$

omitting additive constants. The asymptotic behavior of $\mathcal{D}_{>}^{00}(x)$ is

$$
\mathcal{D}_{>}^{00}(x) \rightarrow \frac{i T}{4 \pi r}+\mathcal{O}\left(e^{-2 \pi T r}\right) .
$$

The leading term, being independent of $\xi$, coincides with the Feynman gauge result. It was only this leading term that contributed to the calculation of the Coulomb potential in Sec II D. Of the remaining components, $\mathcal{D}_{>}^{0 j}(x)$ is exponentially small:

$$
\mathcal{D}_{>}^{0 j}(x)=(1-\xi) \frac{\partial^{2} G_{>}(x)}{\partial t \partial x_{j}} \rightarrow \mathcal{O}\left(e^{-2 \pi T r}\right) .
$$

For the spatial components of the propagator, the term in $d_{>}(x)$ that is linear in $r$ contributes a term from

$$
\frac{\partial^{2}}{\partial x_{i} \partial x_{j}} r=\frac{1}{r}\left(\delta^{i j}-\hat{x}^{i} \hat{x}^{j}\right) .
$$

The asymptotic form of the spatial propagator is

$$
\mathcal{D}_{>}^{i j}(x) \rightarrow \frac{i T}{8 \pi r}\left[-(\xi+1) \delta^{i j}+(\xi-1) \hat{x}^{i} \hat{x}^{j}\right]+\mathcal{O}\left(e^{-2 \pi T r}\right)
$$

Zero-temperature limit: At zero temperature, $d_{>}(x)$ is proportional to $\ln \left[r^{2}-t^{2}\right]$ and Eq. (3.7) gives

$$
\left.\mathcal{D}_{>}^{\mu \nu}(x)\right|_{T=0}=-(\xi+1) \frac{i}{8 \pi^{2}} \frac{g^{\mu \nu}}{x^{2}}+(\xi-1) \frac{i}{4 \pi^{2}} \frac{x^{\mu} x^{\nu}}{\left(x^{2}\right)^{2}} .
$$

The combination $\left.x_{\mu} x_{\nu} \mathcal{D}_{>}^{\mu \nu}(x)\right|_{T=0}=i(\xi-3) / 8 \pi^{2}$ and thus vanishes in the Yennie gauge.

\section{IV. $\mathcal{D}_{>}^{i j}(x)$ IN COULOMB GAUGE}

In the Coulomb gauge the time-like component of the gauge potential is instantaneous and does not propagate in time. Consequently

$$
\mathcal{D}^{00}(x)=\frac{\delta(t)}{4 \pi r},
$$

and there are no thermal corrections. For transverse gauge bosons the zero-temperature propagator is

$$
D_{F}^{i j}(K)=\left(\delta^{i j}-\frac{k^{i} k^{j}}{k^{2}}\right) \frac{1}{K^{2}+i \epsilon} .
$$

and the transverse spectral function is

$$
\rho^{i j}(K)=2 \pi \epsilon\left(k_{0}\right) \delta\left(K^{2}\right)\left[\delta^{i j}-\hat{k}^{i} \hat{k}^{j}\right] .
$$


Using Eq. (1.7), the two-point function can be written

$$
\mathcal{D}_{>}^{i j}(x)=\delta^{i j} \mathcal{D}_{>}(x)+\nabla^{i} \nabla^{j} H_{>}(x),
$$

where the first term, $\mathcal{D}_{>}(x)$, is the same as in Sec. II and the new function is

$$
H_{>}(x)=-i \int \frac{d^{4} K}{(2 \pi)^{3}} \frac{e^{-i K \cdot x}}{1-e^{-\beta k_{0}}} \frac{\epsilon\left(k_{0}\right) \delta\left(K^{2}\right)}{k^{2}} .
$$

By construction, $\mathcal{D}_{>}(x)=-\nabla^{2} H_{>}(x)$.

To compute $H_{>}(x)$ the first step is to integrate over $k_{0}$ and the angles to obtain

$$
H_{>}(x)=\frac{-1}{8 \pi^{2}} \int_{0}^{\infty} \frac{d k}{k^{2}} h_{k}(t, r),
$$

where

$$
h_{k}(t, r)=\frac{1}{r}\left[e^{i k r}-e^{-i k r}\right]\left[\frac{e^{-i k t}}{1-e^{-\beta k}}-\frac{e^{i k t}}{1-e^{\beta k}}\right] .
$$

For later reference note that $H_{>}(x)$ is analytic for complex time in the open region $-\beta<\operatorname{Im} t<0$.

The function $h_{k}(t, r)$ equals $4 i T$ at $k=0$. Consequently the integral over $k$ does not converge at $k=0$. However $\partial H_{>}(x) / \partial r$ is convergent, which is all that is necessary for Eq. (4.1). To improve the $k=0$ behavior it is convenient to subtract an $x$-independent constant from Eq. (4.2) and redefine $H_{>}(x)$ as

$$
H_{>}(x)=\frac{-1}{8 \pi^{2}} \int_{0}^{\infty} \frac{d k}{k^{2}}\left[h_{k}(t, r)-h_{k}(0,0) \frac{\mu^{2}}{k^{2}+\mu^{2}}\right],
$$

where $\mu$ is an unimportant mass parameter. The integral is now convergent at $k=0$ and at $k=\infty$. Since the integrand is even in $k$, the range may be extended over negative $k$ :

$$
H_{>}(x)=\frac{-1}{16 \pi^{2}} \int_{-\infty}^{\infty} \frac{d k}{k^{2}}\left[h_{k}(t, r)-h_{k}(0,0) \frac{\mu^{2}}{k^{2}+\mu^{2}}\right] .
$$

This will now be calculated explicitly for real $t$, without the regularization that was performed in Sec II.

Time-like region: When $t>r$ the contour for the $e^{-i k t}$ contribution may may be closed in the lower halfplane and that for the $e^{i k t}$ contribution closed in the upper half-plane. Although the full integrand has no poles at $k=0$, each of these pieces has a double pole at $k=0$. The more important contribution comes from the simple poles at $k= \pm i 2 \pi n T$ for $n \geq 1$. The result is

$$
H_{>}(x)=\frac{-i}{16 \pi^{3} r T}\left[\operatorname{Li}_{2}\left(z_{-}\right)-\operatorname{Li}_{2}\left(z_{+}\right)\right]+\frac{i}{4 \pi} T t-\frac{1}{8 \pi} .
$$

The last two terms in come from the double pole at $k=0$ and do not contribute to $\partial H_{>}(x) / \partial r$. The variables $z_{ \pm}$ are given in Eq. (2.6) and $\mathrm{Li}_{2}$ is the dilogarithm function, which for $z \leq 1$ has the series expansion [17,18]

$$
\operatorname{Li}_{2}(z) \equiv \sum_{n=1}^{\infty} \frac{z^{n}}{n^{2}}
$$

When $H_{>}(x)$ is substituted into Eq. (4.1) the result is

$$
\mathcal{D}_{>}^{i j}(x)=\left(\delta^{i j}-\hat{x}^{i} \hat{x}^{j}\right) \mathcal{D}_{>}(x)+\left(\delta^{i j}-3 \hat{x}^{i} \hat{x}^{j}\right) E(x)
$$

where the new function $E(x)$ is

$$
\begin{aligned}
E(x)= & \frac{i}{8 \pi^{2} r^{2}}\left[\ln \left(1-z_{-}\right)+\ln \left(1-z_{+}\right)\right] \\
& +\frac{i}{16 \pi^{3} r^{3} T}\left[\operatorname{Li}_{2}\left(z_{-}\right)-\operatorname{Li}_{2}\left(z_{+}\right)\right] .
\end{aligned}
$$

In the deep time-like region, $\pi T(|t|-r) \gg 1$, both $z_{+}$ and $z_{-}$are exponentially small and so is $E(x)$. Since $\mathcal{D}_{>}(x)$ vanishes exponentially as shown in Eq. (2.9), this means that all terms in the Wightman function $\mathcal{D}_{>}^{i j}(x)$ fall exponentially in the deep time-like region.

Space-like region: When $r>|t|$ it is best to return to the defining integral Eq. (4.3). In that integration, the contour for the $e^{i k r}$ contribution may may be closed in the upper half-plane and the contour for the $e^{-i k r}$ contribution closed in the lower half-plane. As previously noted the full integrand has no poles at $k=0$. However each of these pieces has a triple pole at $k=0$. The simple poles at $k= \pm i 2 \pi n T$ give infinite series:

$$
\begin{aligned}
H_{>}(x)= & \frac{i}{16 \pi^{3} r T}\left[\operatorname{Li}_{2}\left(\frac{1}{z_{-}}\right)+\operatorname{Li}_{2}\left(z_{+}\right)\right] \\
& +\frac{i}{8 \pi}\left[T\left(r+\frac{t^{2}}{r}\right)+i \frac{t}{r}-\frac{1}{6 r T}\right] .
\end{aligned}
$$

The last term proportional to $1 / r T$ is particularly unusual.

As mentioned previously, the original integral is analytic in complex time provided $-\beta<\operatorname{Im} t<0$. However it is not obvious that Eq. (4.8) is the analytic continuation of Eq. (4.4), and this provides an important check. In both results the arguments of the dilogarithm functions are smaller than one. For $|z|<1$ the dilogarithm has the integral representation [17,18]

$$
\operatorname{Li}_{2}(z)=\int_{0}^{1} d s(\ln s) \frac{1}{s-z^{-1}} . \quad(|z|<1)
$$

When the time is made complex by $t \rightarrow t-i \sigma$ then $z_{ \pm}=$ $e^{i 2 \pi T \sigma}\left|z_{ \pm}\right|$. Thus if $0<\sigma<\beta / 2$ then $z_{ \pm}$has a positive imaginary part. For $|z|>1$ the analytic continuation is

$$
\operatorname{Li}_{2}(z)=\int_{0}^{1} d s(\ln s) \frac{\mathcal{P}}{s-z^{-1}}+i \pi \ln (z) \quad\left\{\begin{array}{l}
|z|>1 \\
\operatorname{Arg}(z)>0
\end{array} .\right.
$$

Omission of the imaginary part, as is usually done in textbooks, would prevent the function from being analytic. From the integral representation it follows that for $|z|>1$ and $\operatorname{Arg}(z)>0$ the dilogarithm satisfies

$$
\mathrm{Li}_{2}(z)=\frac{\pi^{2}}{3}-\frac{1}{2}(\ln z)^{2}-\mathrm{Li}_{2}\left(\frac{1}{z}\right)+i \pi \ln (z) .
$$


Using this relation one can analytically continue Eq. (4.4) from the time-like region where $z_{-}<1$ to the spacelike region where $z_{-}>1$ and obtain Eq. (4.8). Thus the two results agree despite their appearance.

The Wightman function in the space-like region results from substituting Eq. (4.8) into Eq. (4.1):

$$
\mathcal{D}_{>}^{i j}(x)=\left(\delta^{i j}-\hat{x}^{i} \hat{x}^{j}\right) \mathcal{D}_{>}(x)+\left(\delta^{i j}-3 \hat{x}^{i} \hat{x}^{j}\right) E(x),
$$

where $E(x)$ is now given by

$$
\begin{aligned}
E(x)= & \frac{i T}{8 \pi r}-\frac{i}{8 \pi r^{3}}\left(T t^{2}+i t-\frac{1}{6 T}\right) \\
& +\frac{i}{8 \pi^{2} r^{2}}\left[\ln \left(1-\frac{1}{z_{-}}\right)+\ln \left(1-z_{+}\right)\right] \\
& -\frac{i}{16 \pi^{3} r^{3} T}\left[\operatorname{Li}_{2}\left(\frac{1}{z_{-}}\right)+\operatorname{Li}_{2}\left(z_{+}\right)\right] .
\end{aligned}
$$

Naturally this is the analytic continuation of Eq. (4.7). It is easy to check that $\nabla_{i} \mathcal{D}_{>}^{i j}(x)=0$.

In the deep space-like region defined by $\pi T(r-|t|) \gg 1$, both $1 / z_{-}$and $z_{+}$are exponentially small. The asymptotic behavior of the Wightman function is

$$
\begin{aligned}
\mathcal{D}_{>}^{i j}(x) & \rightarrow\left(\delta^{i j}+\hat{x}^{i} \hat{x}^{j}\right)\left(\frac{-i T}{8 \pi r}\right) \\
& +\left(\delta^{i j}-3 \hat{x}^{i} \hat{x}^{j}\right)\left(\frac{-i}{8 \pi r^{3}}\right)\left(T t^{2}+i t-\frac{1}{6 T}\right) .
\end{aligned}
$$

Despite its complicated appearance this still satisfies the transverse condition $\nabla_{i} \mathcal{D}_{>}^{i j}(x)=0$. The result is more complicated than in covariant gauges, which behaved as $T / r$ with exponentially small corrections. Here the corrections are powers of $r$ : the second line being order $1 / r^{3}$ at fixed $t$ but order $1 / r$ if the ratio $t / r$ is fixed as $r \rightarrow \infty$.

Zero-temperature limit: It is straightforward to evaluate the $T=0$ limit Coulomb-gauge Wightman function. As $T \rightarrow 0$ the arguments of the dilogarithm functions in Eq. (4.7) approach unity: $z \rightarrow 1$. A useful expansion in this region is 17, 18

$$
\operatorname{Li}_{2}(z)=\frac{\pi^{2}}{6}-\ln (z) \ln (1-z)-\sum_{n=1}^{\infty} \frac{(1-z)^{n}}{n^{2}} .
$$

The Wightman function becomes

$$
\begin{aligned}
\left.\mathcal{D}_{>}^{i j}\right|_{T=0} & =\left(\delta^{i j}-\hat{x}^{i} \hat{x}^{j}\right) \frac{i}{4 \pi^{2}\left(t^{2}-r^{2}\right)} \\
& +\left(\delta^{i j}-3 \hat{x}^{i} \hat{x}^{j}\right) \frac{i}{8 \pi^{2}}\left(\frac{2}{r^{2}}-\frac{t}{r^{3}} \ln \left[\frac{t+r}{t-r}\right]\right) .
\end{aligned}
$$

Naturally this agrees with the direct Fourier transform of the zero-temperature propagator.

\section{DISCUSSION}

The thermal Wightman function for free gauge bosons has been computed in various gauges. Knowing the
Wightman function is the same as knowing the timeordered propagators as shown in Appendix A. (The free retarded and advanced propagators are unchanged by the temperature since the free-field commutator is a cnumber and thermal averaging does not change the cnumber.)

The rather surprising result is that the large-distance effects of massless gauge bosons are simpler at $T>0$ than in vacuum. In vacuum the free Green functions fall like $1 /\left(t^{2}-r^{2}\right)$. The thermal Green functions in covariant gauges and in the Coulomb gauge are exponentially small at large time-like separations. At large space-like separations the leading behavior is $T / r$, with exponentially small corrections in covariant gauges and power-law corrections in the Coulomb gauge. Appendix B computes the Wightman functions for the Landshoff-Rebhan approach to thermalization [14].

Since the calculations presented all involve the BoseEinstein distribution function, it is not apparent how much the asymptotic behavior depends on that particular distribution function. The three examples below with different statistics will show that the results obtained in Secs II-IV are a consequence of quantum Bose statistics.

Bose-Einstein Statistics: For Bose-Einstein statistics the simplest case is that of a massless scalar boson. The time-ordered propagator in momentum-space is

$$
D_{11}(K)=\frac{1}{K^{2}+i \eta}-\frac{2 \pi i \delta\left(K^{2}\right)}{e^{\beta\left|k_{0}\right|}-1} .
$$

The Fourier transform of this has been computed in the Feynman gauge discussion Sec II. For real times not on the light cone, a useful representation is that of Eq. (2.8):

$$
\mathcal{D}_{>}(x)=\frac{-i T}{4 \pi r}[\operatorname{coth}(\pi T(r+t))+\operatorname{coth}(\pi T(r-t))] .
$$

The asymptotic behavior as $r \rightarrow \infty$ can be understood by considering the propagator in the Matsubara formulation, which has discrete frequencies $\omega_{n}=2 n \pi T$ because of the periodicity condition in imaginary time [10 12]. As observed by Linde 19.20 the $n=0$ modes produce the dominant behavior in the deep space-like region. The $n=0$ mode contribution is exactly $-i T /(4 \pi r)$. However the large time-like behavior does not come from a single mode in the Matsubara formalism, but requires summing a series and analytically continuing from complex time to real time. The complete result is the same as Eq. (5.2).

Fermi-Dirac Statistics: A convenient way to display the role of statistics is to change the distribution function in Eq. (5.1) from Bose-Einstein to Fermi-Dirac:

$$
D_{11}(K)=\frac{1}{K^{2}+i \eta}+\frac{2 \pi i \delta\left(K^{2}\right)}{e^{\beta\left|k_{0}\right|}+1} .
$$

This differs from Eq. (5.1) in that the -1 in the denominator has been changed to $\mathrm{a}+1$ and also the overall sign of the second term has been changed. This is done so that the propagator for a massless spin $1 / 2$ fermion is 
the product of Eq. (5.3) with $\gamma^{\mu} K_{\mu} 10$ 12. The Fourier transform of Eq. (5.3) can be easily computed along the lines indicated in Sec II. For real times not on the light cone, the result is

$$
\mathcal{D}_{>}(x)=\frac{-i T}{4 \pi r}\left[\frac{1}{\sinh (\pi T(r+t))}+\frac{1}{\sinh (\pi T(r-t))}\right] .
$$

At zero temperature this is the same as for Bose-Einstein statistics. At large time-like separations it falls exponentially as in the Bose-Einstein case. At large spacelike separations it also falls exponentially in contrast to Eq. (5.2). The space-like behavior can be understood in the Matsubara formalism, since the fermion frequencies $\omega_{n}=(2 n+1) \pi T$ can never vanish.

Classical Statistics: Classical Boltzman statistics gives a very different result. In the classical limit the thermal distribution function in Eq. (5.1) is no longer singular at $k_{0}=0$ :

$$
D_{11}(K)=\frac{1}{K^{2}+i \eta}-\frac{2 \pi i \delta\left(K^{2}\right)}{e^{\beta\left|k_{0}\right|}} .
$$

The Fourier transform of this gives

$\mathcal{D}_{>}(x)=\frac{-i}{4 \pi^{2}}\left[\frac{1}{r^{2}-(t-i \epsilon)^{2}}+\frac{1}{r^{2}-(t-i \beta)^{2}}+\frac{1}{r^{2}-(t+i \beta)^{2}}\right]$

At zero temperature this is the same as the Bose or Fermi case. However at $T \neq 0$ it is quite different. For large time-like separations it falls like $1 / t^{2}$. For large spacelike separations it also falls like $1 / r^{2}$. Thus, for classical statistics the temperature does not substantially change the asymptotic behavior.

Subsequent publications will explore the physical consequences of the space-time behavior. It should be possible to understand the hard thermal loop approximation [15] directly in coordinate space. In that approximation the high temperature corrections come entirely from one-loop diagrams. The number of external lines determines the number of internal propagators. The important effects come from thermal corrections on one internal propagator with all others kept at their zerotemperature value. A coordinate-space analysis should explain why only one loop diagrams are important and why only one propagtor in the loop enjoys thermal corrections. In addition, since non-equilibrium processes almost demand a coordinant space treatment, it may be possible to deduce an extension of the hard loop approximation to non-equilibrium processes.

\section{ACKNOWLEDGMENTS}

This work was supported in part by the U.S. National Science Foundation under grant PHY-9900609.

\section{APPENDIX A: GENERAL STRUCTURE}

This appendix summarizes some standard properties of gauge boson propagators at finite temperature $10-12$. The basic thermal Wightman function is

$$
\mathcal{D}_{>}^{\mu \nu}(x)=-i \sum_{n} e^{-\beta E_{n}} \frac{\left\langle n\left|A^{\mu}(x) A^{\nu}(0)\right| n\right\rangle}{\operatorname{Tr}\left[e^{-\beta H}\right]} .
$$

It is customary to introduce a special notation $\mathcal{D}_{<}(x)$ for the function with inverted space-time arguments:

$$
\mathcal{D}_{<}^{\mu \nu}(x) \equiv \mathcal{D}_{>}^{\nu \mu}(-x)
$$

In terms of the field operators this means that

$$
\mathcal{D}_{<}^{\mu \nu}(x)=-i \sum_{n} e^{-\beta E_{n}} \frac{\left\langle n\left|A^{\nu}(0) A^{\mu}(x)\right| n\right\rangle}{\operatorname{Tr}\left[e^{-\beta H}\right]} .
$$

The retarded and advanced propagators are given by

$$
\begin{gathered}
\mathcal{D}_{R}^{\mu \nu}(x)=\theta(t)\left[\mathcal{D}_{>}^{\mu \nu}(x)-\mathcal{D}_{<}^{\mu \nu}(x)\right] \\
\mathcal{D}_{A}^{\mu \nu}(x)=\theta(-t)\left[\mathcal{D}_{<}^{\mu \nu}(x)-\mathcal{D}_{>}^{\mu \nu}(x)\right] .
\end{gathered}
$$

For any parameter $\sigma$ in the range $0 \leq \sigma \leq \beta$, the four parts of the contour-ordered propagator are

$$
\begin{aligned}
& \mathcal{D}_{11}^{\mu \nu}(x)=\theta(t) \mathcal{D}_{>}^{\mu \nu}(x)+\theta(-t) \mathcal{D}_{<}^{\mu \nu}(x) \\
& \mathcal{D}_{12}^{\mu \nu}(x)=\mathcal{D}_{<}^{\mu \nu}(t+i \sigma, \vec{x}) \\
& \mathcal{D}_{21}^{\mu \nu}(x)=\mathcal{D}_{>}^{\mu \nu}(t-i \sigma, \vec{x}) \\
& \mathcal{D}_{22}^{\mu \nu}(x)=\theta(t) \mathcal{D}_{<}^{\mu \nu}(x)+\theta(-t) \mathcal{D}_{>}^{\mu \nu}(x) .
\end{aligned}
$$

With respect to real time, the first of these is timeordered; the fourth is anti-time-ordered.

The spectral function is the thermal average of the commutator

$$
\rho^{\mu \nu}(x)=\sum_{n} e^{-\beta E_{n}} \frac{\left\langle n\left|\left[A^{\mu}(x), A^{\nu}(0)\right]\right| n\right\rangle}{\operatorname{Tr}\left[e^{-\beta H}\right]} .
$$

Its Fourier transform $\rho^{\mu \nu}(K)$ has the properties

$$
\begin{aligned}
\rho^{\mu \nu}(-K) & =-\rho^{\nu \mu}(K) \\
\rho^{\mu \nu}(K)^{*} & =\rho^{\nu \mu}(K) .
\end{aligned}
$$

The Fourier transform of Eq. (A1) is

$$
D_{>}^{\mu \nu}(K)=-i \frac{\rho^{\mu \nu}(K)}{1-e^{-\beta k_{0}}} .
$$

This is the starting point in Eq. (1.6). The spectral function is most easily obtained from the propagator in momentum space by the relation

$$
\rho^{\mu \nu}(K)=i\left[D_{R}^{\mu \nu}(K)-D_{A}^{\mu \nu}(K)\right] .
$$




\section{APPENDIX B: LANDSHOFF-REBHAN PROPAGATOR}

Landshoff and Rebhan have advocated heating only the two physical (i.e. spatially transverse) components of the gauge potential even in a general covariant gauge [14]. The procedure is to begin with the $T=0$ propagator in a general covariant gauge,

$$
D_{11}^{\mu \nu}(K)=\frac{-g^{\mu \nu}}{K^{2}+i \epsilon}+(1-\xi) \frac{K^{\mu} K^{\nu}}{\left(K^{2}+i \epsilon\right)^{2}} .
$$

As explained in [14], the $D^{00}(K)$ and $D^{0 j}(K)$ components are unaffected by temperature and therefore the corresponding Wightman functions in space-time may be read off from Eq. (3.9):

$$
\begin{aligned}
& \mathcal{D}_{>}^{00}(x)=\frac{i}{8 \pi^{2}} \frac{(\xi-3) t^{2}+(\xi+1) r^{2}}{\left(x^{2}-i \eta\right)^{2}} \\
& \mathcal{D}_{>}^{0 j}(x)=\frac{i}{4 \pi^{2}}(\xi-1) \frac{t x^{j}}{\left(x^{2}-i \eta\right)^{2}} .
\end{aligned}
$$

To separate the physical components of $\vec{A}$, rearrange the spatial components of the $T=0$ propagator as

$$
D_{11}^{i j}(K)=\frac{\delta^{i j}-k^{i} k^{j} / k^{2}}{K^{2}+i \epsilon}+\frac{\left(k_{0}^{2}-\xi k^{2}\right) k^{i} k^{j} / k^{2}}{\left(K^{2}+i \epsilon\right)^{2}} .
$$

The first term describes physical, transversely polarized, particles and is heated. The second term comes from gauge-dependent, longitudinally polarized particles and is not heated. From the previous results the finitetemperature two point function is

$$
\mathcal{D}_{>}^{i j}(x)=\mathcal{D}_{>\text {Coul }}^{i j}(x)+F_{>}^{i j}(x)
$$

where the temperature-dependent Coulomb part is given by Eqs. (4.6), (4.7) in the time-like region and by Eqns. (4.10), (4.11) in the space-like region. The new temperature-independent contribution $F_{>}^{i j}(x)$ is

$$
\begin{aligned}
F_{>}^{i j}(x) & =\left(\delta^{i j}-\hat{x}^{i} \hat{x}^{j}\right) \frac{i}{16 \pi^{2}} \frac{\xi+1}{t^{2}-r^{2}}+\hat{x}^{i} \hat{x}^{j} \frac{i}{4 \pi^{2}} \frac{t^{2}}{\left(t^{2}-r^{2}\right)^{2}} \\
& +\left(\delta^{i j}-3 \hat{x}^{i} \hat{x}^{j}\right) \frac{i}{16 \pi^{2}}\left(\frac{\xi-3}{t^{2}-r^{2}}-\frac{4}{r^{2}}+\frac{2 t}{r^{3}} \ln \left[\frac{t+r}{t-r}\right]\right) .
\end{aligned}
$$

The Landshoff-Rebhan propagator clearly has a very complicated asymptotic behavior. In the deep space-like region, $r \rightarrow \infty$, Eqs. (B1) fall like $1 / r^{2}$, Eq. (B2) falls like $1 / r^{3}$, whereas the Coulomb part of Eq. (B3) still falls like $1 / r$. In the deep time-like region, $t \rightarrow \infty$, Eqns. (B1), (B2), and (B3) are all dominated by the zero-temperature contributions and fall like $1 / t^{2}$ rather than exponentially.

[1] J. Schwinger, J. Math. Phys. 2, 407 (1961).
[2] L. Kadanoff and G. Baym, Quantum Statistical Mechanics, (Benjamin, New York, 1962).

[3] L.V. Keldysh, Sov. Phys. JETP 20, 1018 (1964).

[4] E.M. Lifshitz and L.P. Piteevskii, Physical Kinetics, (Pergamon Press, Oxford, 1981).

[5] K.C. Chou, Z.B. Su, B.L. Hao, and L.Yu, Phys. Rep. 118, 1 (1985).

[6] U. Heinz, Phys. Rev. Lett. 51, 351 (1983); Ann. Phys. (N.Y.) 161, 48 (1985).

[7] S. Mrówczyński and U. Heinz, Ann. Phys. (N.Y.) 229, 1 (1994).

[8] P. Danielewicz, Ann. Phys. 229, 1 (1994).

[9] J.P. Blaizot and E. Iancu, Nucl. Phys. B557, 193 (1999).

[10] N.P. Landsman and Ch. G. van Weert, Phys. Rep. 145, 141 (1987).

[11] M. Le Bellac, Thermal Field Theory (Cambridge University Press, Cambridge, England, 1996).

[12] A. Das, Finite Temperature Field Theory (World Scientific, Singapore, 1997).

[13] R. Kubo, J. Phys. Soc. Japan 12, 570 (1957); P. Martin and J. Schwinger, Phys. Rev. 115, 1342 (1959).

[14] P.V. Landshoff and A. Rebhan, Nucl. Phys. B383, 607 (1992) and B410, 23 (1993).

[15] E. Braaten and R.D. Pisarski, Nucl. Phys. B337, 569 (1990) and B339, 310 (1990).

[16] I.S. Gradshteyn and I.M. Ryzhik, Table of Integrals, Series, and Products (Academic Press, New York, 1980).

[17] M. Abramowitz and I.A. Stegun, Handbook of Mathematical Functions (National Bureau of Standards, Washington, D.C., 1964).

[18] L. Lewin, Dilogarithms and Associated Functions (MacDonald, London, 1958).

[19] A.D. Linde, Phys. Lett. B96, 289 (1980).

[20] D. J. Gross, R.D. Pisarski, and L.G. Yaffe, Rev. Mod. Phys. 53, 43 (1981). 